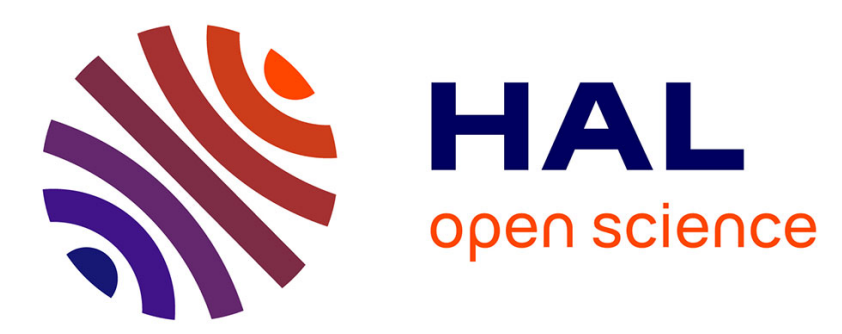

\title{
Influence de la température ambiante sur les performances de croissance du porc
}

\author{
Dominique Rinaldo, J. Le Dividich
}

\section{To cite this version:}

Dominique Rinaldo, J. Le Dividich. Influence de la température ambiante sur les performances de croissance du porc. Productions Animales, 1991, 4 (1), pp.57-65. hal-00895925

\section{HAL Id: hal-00895925 \\ https://hal.science/hal-00895925}

Submitted on 1 Jan 1991

HAL is a multi-disciplinary open access archive for the deposit and dissemination of scientific research documents, whether they are published or not. The documents may come from teaching and research institutions in France or abroad, or from public or private research centers.
L'archive ouverte pluridisciplinaire HAL, est destinée au dépôt et à la diffusion de documents scientifiques de niveau recherche, publiés ou non, émanant des établissements d'enseignement et de recherche français ou étrangers, des laboratoires publics ou privés. 
INRA Prod. Anim., 1991, 4 (1), $57-65$
Dominique RINALDO et J. LE DIVIDICH

INRA Station de Recherches Porcines Saint-Gilles 35590 L'Hermitage

\section{Influence} de la température ambiante sur les performances de croissance du porc

Les performances du porc en croissance-finition dépendent largement des conditions thermiques offertes à l'animal, mais il est difficile d'optimiser simultanément tous les critères de production. Une température ambiante trop basse n'est pas limitante pour la croissance mais le coût alimentaire est nécessairement augmenté. En élevage intensif sur caillebotis, une température de $24-25^{\circ} \mathrm{C}$ réduit à la fois l'indice de consommation et l'adiposité des carcasses.

$\mathrm{Au}$ cours de ces dernières décennies, l'intensification de la production porcine s'est manifestée par une augmentation de la taille des ateliers, de la densité d'animaux dans les bâtiments et la généralisation de l'élevage du porc en croissance sur sol en caillebotis. Parallèlement, la réduction de l'état d'engraissement,

\section{Résumé}

L'objectif de cet article est de préciser les effets de la température ambiante sur le métabolisme énergétique, les performances de croissance et les besoins nutritionnels du porc en croissance-finition en élevage intensif et d'estimer son optimum thermique. Au plan énergétique, la température critique est estimée à environ $20^{\circ} \mathrm{C}$ en croissance et $15{ }^{\circ} \mathrm{C}$ en finition. Elle correspond à une utilisation maximale de l'énergie alimentaire et sa signification zootechnique est discutée. La température ambiante influence la nature des dépôts tissulaires, l'accrétion lipidique étant la plus affectée. Pour ce qui est des performances, en alimentation libérale, la consommation spontanée d'aliment d'un porc de $60 \mathrm{~kg}$ diminue de $22 \mathrm{~g} / \mathrm{j} /{ }^{\circ} \mathrm{C}$ entre 10 et $20^{\circ} \mathrm{C}$, sans effet sur le gain de poids. Entre 20 et $30^{\circ} \mathrm{C}$, cette diminution est plus marquée $\left(73 \mathrm{~g} / \mathrm{j} /{ }^{\circ} \mathrm{C}\right)$ et s'accompagne d'une réduction du gain de poids $\left(40 \mathrm{~g} / \mathrm{j} /{ }^{\circ} \mathrm{C}\right)$ et de l'adiposité corporelle. L'indice de consommation décroît de 0,044 unité $/{ }^{\circ} \mathrm{C}$ entre 10 et $20^{\circ} \mathrm{C}$ et est minimal vers $25^{\circ} \mathrm{C}$. En tenant compte des objectifs de réduction du coût alimentaire et de l'état d'engraissement des carcasses, la température optimale pour le porc en croissance-finition élevé sur caillebotis béton est de $24-25^{\circ} \mathrm{C}$. Les études d'interaction avec l'alimentation ont montré qu'au froid les animaux valorisent bien les aliments riches en fibres alors qu'au chaud les meilleures performances sont obtenues avec des rations concentrées en énergie. Ces études ont également permis d'estimer l'accroissement du besoin énergétique à $25 \mathrm{~kJ} \mathrm{EM} / \mathrm{kg}^{0,75} / \mathrm{j}^{\circ} \mathrm{C}$ entre 20 et $12^{\circ} \mathrm{C}$ et de montrer que, pour un même gain de poids ou de muscle, le besoin journalier en acides aminés est indépendant de la température ambiante. par voie génétique (sélection, croisement) et par une meilleure adéquation des apports nutritionnels aux besoins, a accentué la sensibilité du porc à son environnement thermique. L'optimisation des performances de croissance et de l'utilisation des aliments sont ainsi, de plus en plus, tributaires des conditions thermiques offertes à l'animal. Elles déterminent en effet, pour une large part, l'importance des échanges de chaleur entre l'animal et son milieu environnant et par conséquent le devenir de l'énergie alimentaire. Par ailleurs, elles ont une influence directe sur le niveau de consommation spontanée et, de ce fait, sont susceptibles de modifier les équilibres nutritionnels.

Plusieurs revues bibliographiques ont été consacrées aux effets de l'environnement thermique sur le métabolisme énergétique (Close 1981) et l'état sanitaire (Thomas 1984) du porc. En revanche, les effets sur les performances de croissance sont moins connus (Verstegen et al 1978). L'objectif de cet article est précisément de faire le point des acquisitions récentes sur l'influence de l'environnement thermique et notamment de la température ambiante sur les performances du porc en croissance. Après avoir brièvement rappelé l'effet de la température ambiante sur le métabolisme énergétique, nous préciserons son influence sur les performances de croissance et la composition corporelle. Enfin, nous ferons état dés interactions alimentation-milieu climatique. 


\section{1 / Influence de la température ambiante sur le métabolisme énergétique}

\section{1 / Production de chaleur et signification de la température critique}

La relation entre la production de chaleur chez le porc et la température ambiante est schématiquement représentée à la figure 1. La production de chaleur est minimale dans la zone de neutralité thermique limitée par les températures critiques, inférieure (Tci) et supérieure (Tcs). D'un point de vue nutritionnel, il est particulièrement intéressant, dans nos régions à climat tempéré, de connaître la température critique inférieure, plus généralement dénommée température critique. Elle est déterminée par calorimétrie et correspond chez l'animal à une dépense énergétique minimale. D'après les travaux réalisés pour la plupart en Grande-Bretagne (Close 1981) et aux Pays-Bas (Verstegen et al 1978), la température critique dépend de l'animal (poids, âge, niveau alimentaire, taille du groupe) et des paramètres de l'environnement climatique (vitesse de l'air, humidité relative, nature du sol et des parois). Concrètement, en conditions d'alimentation à volonté et d'élevage en groupe sur sol en caillebotis béton partiellement humide, la température critique est de l'ordre de 18 à $20^{\circ} \mathrm{C}$ au cours de la croissance et de 14 à $15^{\circ} \mathrm{C}$ en période de finition (NRC 1981).

Figure 1. Utilisation de l'énergie par le porc de 10 à $30 \mathrm{~kg}$ de poids vif, en relation avec la température ambiante (adapté de Rinaldo 1989).

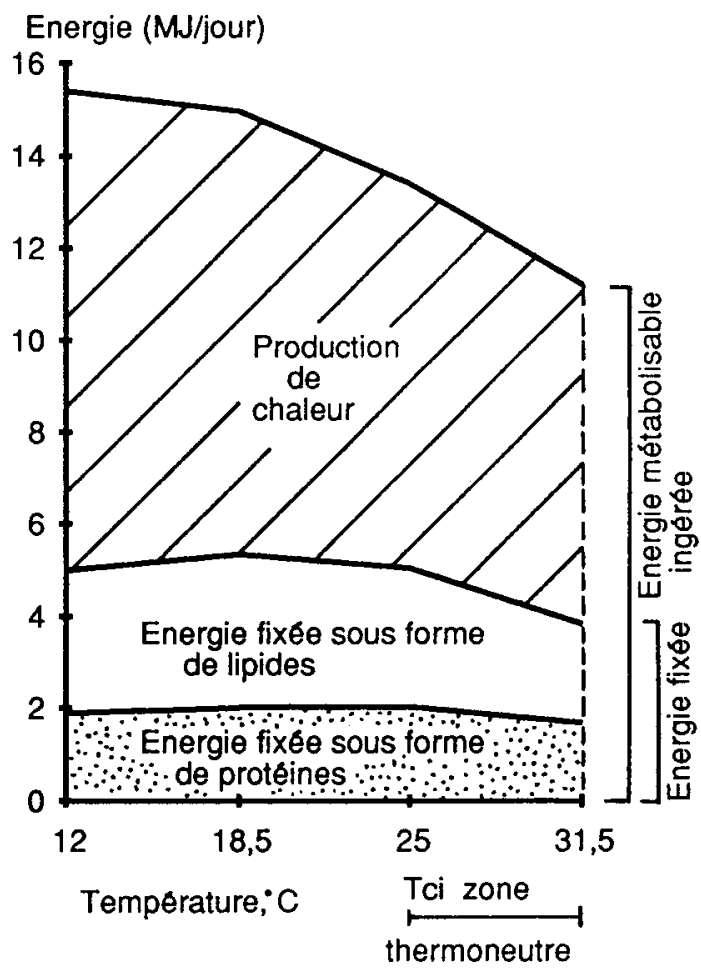

Cependant, la température critique a une signification essentiellement énergétique puisqu'elle représente la température minimale permettant une utilisation maximale de l'énergie alimentaire et, par conséquent, une fixation maximale d'énergie sous forme de protéines et de lipides. Au plan zootechnique, elle correspond au " besoin thermique optimal » du porc à 2 stades où il manifeste une sensibilité particulière au froid: la naissance et la période de sevrage. A ces deux stades, il s'agit en effet de favoriser l'accrétion lipidique, chez le nouveauné pour améliorer rapidement son isolation thermique et, chez le porcelet au stade du sevrage, pour éviter un amaigrissement excessif et, par conséquent, limiter la sensibilité de l'animal au froid. A l'exception de ces 2 périodes, la température critique a une signification zootechnique limitée dans la mesure où une fixation maximale d'énergie ne correspond pas nécessairement à un gain de poids maximal et où l'objectif est de réduire l'adiposité des carcasses. Néanmoins, on peut s'interroger sur les conséquences de la recherche d'une croissance musculaire maximale, par voie génétique ou grâce aux manipulations hormonales, sur la température critique. Dans la mesure où une réduction supplémentaire de l'état d'engraissement ne peut être que marginale chez ces animaux à fort développement musculaire ou traités à l'hormone de croissance, la température critique représente alors une bonne estimation de la température optimale.

\section{2 / Rendement d'utilisation de l'énergie pour la croissance}

L'énergie métabolisable ingérée (EMi) se partitionne en énergie retenue ou fixée (ER) et en énergie perdue sous forme de chaleur et donc improductive, selon l'équation suivante : EMi $=$ ER + Production ou pertes de chaleur

La transformation de l'EMi en ER fait intervenir le rendement $(K)$ d'utilisation de l'EMi pour la croissance. Les résultats obtenus sur porcelets sevrés (Noblet et Le Dividich 1982), sur porc en croissance (Verstegen et al 1973, Close 1978, Noblet et al 1985) et sur truie (Noblet et al 1988) indiquent une augmentation significative de ce rendement en conditions froides (tableau 1). En réalité, cette amélioration traduit une participation de l'extra-chaleur associée à l'utilisation de l'EMi pour les synthèses, à la dépense énergétique de thermorégulation. En d'autres termes, l'extra-chaleur, qui doit être totalement dissipée à la neutralité thermique, couvre une partie de l'accroissement du besoin énergétique provoqué par l'abaissement de la température ambiante. Comme nous le verrons par la suite, ce résultat a été largement utilisé pour tenter de tirer profit/des propriétés thermogéniques des aliments.

\section{3 / Rétention énergétique sous forme de protéines et de lipides}

Sur ce point, les auteurs s'accordent à reconnaître que l'environnement thermique a davantage d'effet sur l'accrétion lipidique que sur le dépôt protéique (figure 2). En alimentation éga- 
Tableau 1. Effet de la température ambiante sur le rendement $(K)$ de transformation de l'énergie métabolisable ingérée en énergie fixée chez le porc en croissance.

\begin{tabular}{|c|c|c|l|}
\hline \multicolumn{3}{|c|}{ Intervalle de température, "C } & \multirow{2}{*}{ Références } \\
\hline $\mathbf{8}-\mathbf{1 3}$ & $\mathbf{2 0}-\mathbf{2 5}$ & $\mathbf{3 0}$ & \\
\hline 0,80 & 0,72 & - & Fuller et Boyne 1972 \\
0,99 & 0,67 & - & Verstegen et al 1973 \\
$\mathbf{0 , 7 9}$ & 0,71 & 0,63 & Close 1978 \\
\hline
\end{tabular}

lisée, l'accrétion lipidique croît avec la température ambiante (Verstegen et al 1973, Close et al 1978, Noblet et Le Dividich 1982), mais, en alimentation à volonté, elle diminue aux fortes températures en raison de l'abaissement du niveau d'ingestion spontanée (Campbell et Taverner 1988, Rinaldo et Le Dividich 1991). En revanche, l'effet de la température ambiante sur le dépôt protéique dépend du stade physiologique de l'animal. Jusqu'au sevrage, ce dépôt est indépendant de la température ambiante même lorsque le porcelet est en bilan énergétique négatif (Le Dividich et al 1980). Toutefois, chez l'animal en croissance-finition, les protéines peuvent être utilisées comme source d'énergie au froid lorsque l'apport énergétique est limitant. Les travaux de Bershauer et al (1983) et de Campbell et Taverner (1988) sont très significatifs à cet égard. Ils montrent qu'en alimentation égalisée, lorsque l'apport énergétique est élevé, soit $1300 \mathrm{~kJ} \mathrm{~d}^{\prime} \mathrm{EM} / \mathrm{kg}^{0,75} / \mathrm{jour}$, l'accrétion protéique ne décroît que de $0,13 \mathrm{~g} / \mathrm{kg}^{0.75} / \mathrm{j}$ et par "C de réduction de température entre 22 et $10^{\circ} \mathrm{C}$, mais elle diminue de $0,26 \mathrm{~g} / \mathrm{kg}^{10,75} / \mathrm{j} /{ }^{\circ} \mathrm{C}$ lorsque le niveau d'ingestion est plus faible $(800 \mathrm{~kJ}$ d'EM/ $\mathrm{kg}^{0.75} / \mathrm{j}$ \}. Ces résultats suggèrent donc que, chez le porc en croissance-finition, l'influence des conditions climatiques sur le métabolisme azoté dépend essentiellement de l'apport énergétique.

\section{2 / Influence de la température ambiante sur les performances de croissance}

\section{1 / Consommation d'aliment}

Le porc, comme tout homéotherme, modifie son niveau d'ingestion alimentaire en réponse aux variations de la température ambiante. Cet ajustement représente un mécanisme essentiel d'adaptation aux conditions climatiques d'élevage (Herpin 1988). Il est rapide puisque, chez le porc acclimaté à $20^{\prime \prime} \mathrm{C}$, la consommation se stabilise en 6 jours lorsqu'on abaisse la température ambiante à $15^{\circ} \mathrm{C}$.

Selon les mesures effectuées par Rinaldo (1989) au cours de la période 10 à $30 \mathrm{~kg}$ de poids vif et par Nienaber et Leroy Hahn (1983) au cours de la période $40-85 \mathrm{~kg}$ de poids vif, la quantité d'aliment spontanément ingéré (Y) diminue de manière curvilinéaire avec l'augmentation de la température ambiante (T) selon les équations suivantes :

- Période 10-30 kg :

$\mathrm{Y}(\mathrm{g} / \mathrm{j})=1163+16,80 \mathrm{~T}-0,82 \mathrm{~T}^{2}\left(\mathrm{R}^{2}=0,73\right)$
- Période 40-85 kg :

$\mathrm{Y}(\mathrm{g} / \mathrm{j})=1520+10,57 \mathrm{P}+54,6 \mathrm{~T}-2,57 \mathrm{~T}^{2}$

$\left(\mathrm{R}^{2}=0,67\right)$

où $\mathrm{P}$ représente le poids vif $(\mathrm{kg})$ de l'animal. Concrètement, chez le porc de $20 \mathrm{~kg}$ de poids vif, la quantité d'aliment consommé diminue d'environ $8 \mathrm{~g} / \mathrm{j}$ et par "C d'augmentation de la température dans l'intervalle $10-20^{\circ} \mathrm{C}$ et de $23 \mathrm{~g} / \mathrm{j} / /^{\prime \prime} \mathrm{C}$, entre 20 et $30^{\circ} \mathrm{C}$. Les valeurs correspondantes pour un porc de $60 \mathrm{~kg}$ de poids vif sont de 22 et $73 \mathrm{~g} / \mathrm{j} /{ }^{\circ} \mathrm{C}$, respectivement.

Le comportement alimentaire est également susceptible de varier avec la température ambiante. Chez le rat, par exemple, l'hyperpha-

Figure 2. Influence de la température ambiante sur la production de chaleur et la partition de l'énergie fixée sous forme de protéines et de lipides selon le mode d'alimentation et le stade physiologique (d'après Le Dividich et al 1980 (A), Verstegen et al 1973, Close et al 1978, Noblet et Le Dividich 1982 (B), Close et al 1978 et Campbell et Taverner $1988(C)$ ).
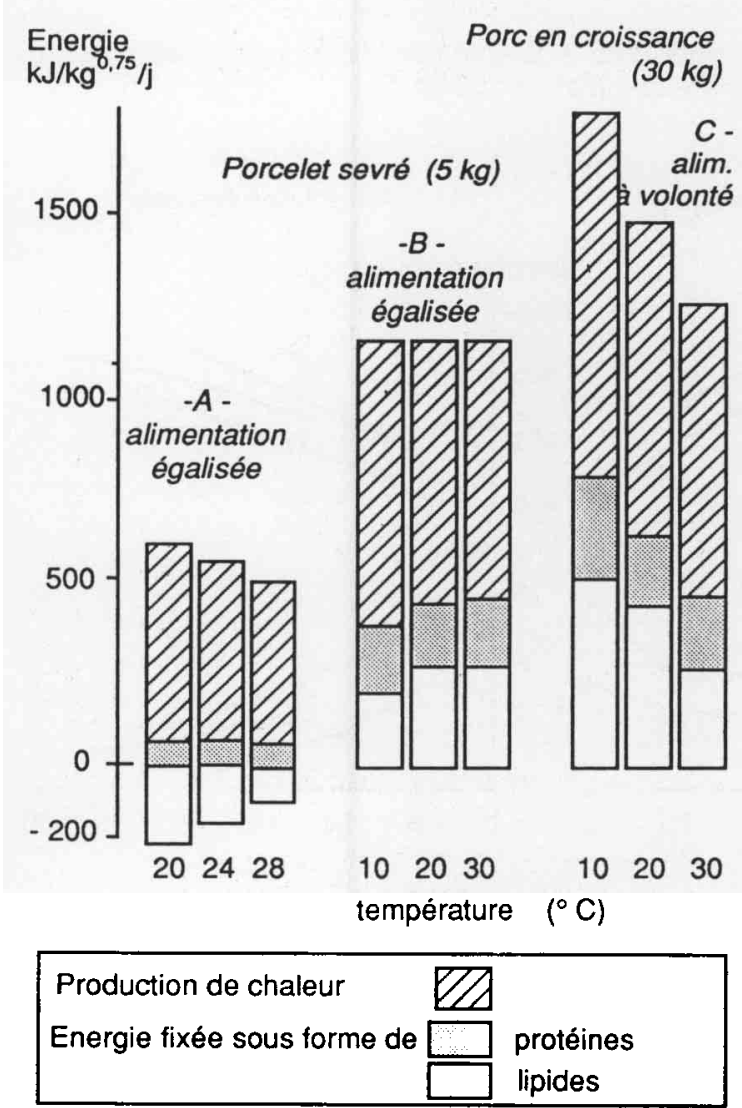

La température ambiante a peu d'effet sur le dépôt protéique. 
gie nocturne s'étend à la phase diurne lors d'une adaptation au froid. Il en résulte que l'extra-chaleur associée aux processus de digestion et de synthèse tissulaire est mieux répartie sur le nycthémère et par conséquent mieux utilisée pour la thermorégulation. Chez le porc adapté au froid, Herpin (1988) n'observe cependant aucune modification de la répartition nycthémérale de la prise alimentaire. En revanche, selon Rinaldo et al (1989) un abaissement nocturne de la température ambiante entraîne une augmentation de la proportion d'aliment consommé au cours de la nuit. Par ailleurs, relativement à des animaux maintenus à température constante élevée $\left(33^{\circ} \mathrm{C}\right)$, ceux soumis à une température cyclique de même moyenne journalière consomment une plus forte proportion d'aliment au cours des périodes fraîches du nycthémère. Quoi qu'il en soit, l'effet de la température ambiante sur le comportement alimentaire est moins net chez le porc que chez d'autres espèces, sans doute, parce que les rythmes biologiques sont naturellement peu marqués dans l'espèce porcine.

\section{2 / Croissance journalière et indice de consommation}

Les performances de croissance de porcs recevant un régime équilibré sont avant tout liées aux quantités d'aliment ingérées, qui, pour une large part, dépendent de la température ambiante. De ce fait, ces performances varient considérablement avec les conditions climatiques selon que le mode d'alimentation est libéral ou restreint (figure 3).

Figure 3. Influence de la température ambiante sur les performances du porc en croissance-finition selon le mode d'alimentation.

Alimentation à volonté

Gain de poids, $g / j$

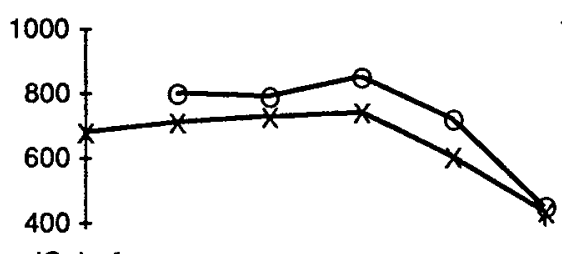

IC, $\mathrm{kg} / \mathrm{kg}$

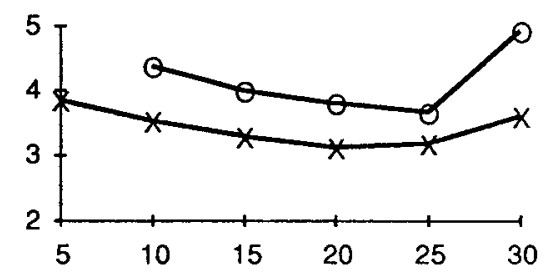

Temperature ambiante, ${ }^{\bullet} \mathrm{C}$
Alimentation égalisée

Gain de poids, $g / j$

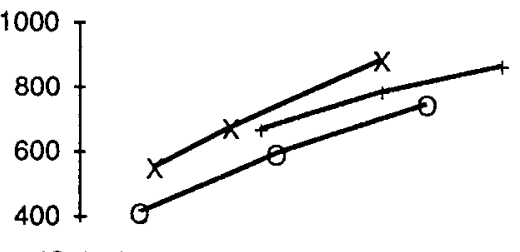

IC, $\mathrm{kg} / \mathrm{kg}$

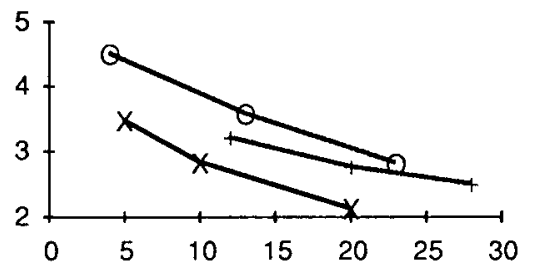

Nichols et al, 1982

Nienaber et Leroy Hahn, 1983
* Bresk et Stolpe, 1982

Fuller et Boyne, 1971

Ledividich et al, 1985
En alimentation à volonté, les recommandations moyennes de température ambiante sont de l'ordre de 20 à $23^{\circ} \mathrm{C}$ au cours de la phase initiale de croissance (entre 10 et $30 \mathrm{~kg}$ de poids vif, Le Dividich et al 1982) et de 18 à $21^{\circ} \mathrm{C}$ au cours de la phase ultérieure (entre 30 et $100 \mathrm{~kg}$ de poids vif, NRC 1981). Cependant, sur une plage de 8 à $10^{\circ} \mathrm{C}$ inférieure à ces recommandations, le gain de poids journalier reste relativement constant chez le porc en croissance tout comme chez l'animal plus âgé. En revanche, aux températures supérieures aux recommandations, il diminue d'environ $15 \mathrm{~g} /{ }^{\circ} \mathrm{C}$ chez le porc en croissance et de $40 \mathrm{~g} /{ }^{\circ} \mathrm{C}$ chez le porc plus lourd.

En alimentation égalisée, la vitesse de croissance augmente avec la température ambiante. L'accroissement est en moyenne de $11 \mathrm{~g} /{ }^{\circ} \mathrm{C}$ entre 18 et $25^{\circ} \mathrm{C}$ chez le porc en croissance et de $15-17 \mathrm{~g} /{ }^{\circ} \mathrm{C}$ entre 5 et $20^{\circ} \mathrm{C}$ chez l'animal plus lourd. Entre 20 et $25-28^{\circ} \mathrm{C}$, la seule donnée existante fait état d'une amélioration de $8 \mathrm{~g} /{ }^{\circ} \mathrm{C}$.

Enfin, l'indice de consommation dépend étroitement de la température ambiante et du mode d'alimentation, à volonté ou égalisé. Entre 30 et $100 \mathrm{~kg}$ de poids vif, il augmente de 0,044 unité en moyenne par degré inférieur aux recommandations lorsque l'alimentation est du type libéral, et de 0,070 unité lorsque l'alimentation est égalisée. La différence, qui s'explique par la réduction de la température critique chez l'animal nourri à volonté, souligne donc la nécessité de tenir compte du niveau alimentaire dans l'estimation de la température optimale.

\section{3 / Composition corporelle}

L’influence de la température ambiante sur la composition corporelle dépend essentiellement, comme pour la vitesse de croissance, du niveau alimentaire. A niveau alimentaire constant, la diminution de la température ambiante a, sur la composition corporelle, un effet comparable à celui d'une restriction alimentaire (tableau 2). A même gain de poids, c'est-à-dire lorsque l'abaissement de la température ambiante est compensé par un apport supplémentaire d'aliment, nos résultats (Le Dividich et al 1987) et ceux de Lefaucheur et al (1989) ne mettent en évidence aucun effet de la température sur les caractéristiques des carcasses. Enfin, en alimentation à volonté, les carcasses sont plus grasses au froid (Nienaber et Leroy Hahn 1983, Verstegen et al 1985, Campbell et Taverner 1988). En revanche, selon Rinaldo et Le Dividich (1991), l'augmentation de la température ambiante dans la zone de thermoneutralité entraîne une réduction importante de l'adiposité des carcasses consécutive à la réduction de la consommation d'aliment. D'après ces auteurs, cette réduction correspond à un rationnement vrai de l'animal dans la mesure où le niveau d'ingestion spontanée diminue de manière importante alors que le besoin énergétique d'entretien reste constant.

L'effet de la température ambiante se manifeste par ailleurs sur la morphologie et la conformation du porc. Ainsi, au froid, les animaux sont plus courts et plus compacts (Lefau- 
Tableau 2. Influence de la température ambiante sur la composition corporelle à l'abattage (base $100=$ résultats obtenus à $20^{\circ} \mathrm{C}$ ) (d'après Le Dividich et al 1985, 1987).

\begin{tabular}{|l|r|r|r|}
\hline \multicolumn{1}{|c|}{ Température ambiante, ${ }^{\circ} \mathbf{C}$} & $\mathbf{1 2}$ & $\mathbf{2 0}$ & $\mathbf{2 8}$ \\
\hline \multicolumn{5}{|c|}{ A même niveau de rationnement } \\
\hline \% muscle & 103 & 100 & 98 \\
\% gras & 91 & 100 & 104 \\
Longe : Bardière & 115 & 100 & 95 \\
\% Bardière & 92 & 100 & 105 \\
\% Panne & 85 & 100 & 117 \\
\hline \multicolumn{3}{|c|}{ A même gain de poids journalier } \\
\hline \% muscle & \multicolumn{3}{|c|}{9} \\
\% gras & 97 & 100 & 101 \\
Longe : Bardière & 103 & 100 & 104 \\
\% Bardière & 88 & 100 & 95 \\
\% Panne & 109 & 100 & 120 \\
\hline
\end{tabular}

Tableau 3. Influence de la température ambiante d'élevage sur le degré d'insaturation des graisses de la bardière.

\begin{tabular}{|l|c|c|c|c|}
\hline \multirow{2}{*}{ Critère } & \multicolumn{2}{|c|}{ Température ambiante, ${ }^{\circ} \mathbf{C}$} & \multirow{2}{*}{ Références } \\
\cline { 2 - 4 } & $\mathbf{1 2 - 1 3}$ & $\mathbf{2 0 - 2 3}$ & $\mathbf{2 8}$ & \\
\hline Indice d'iode & 67,2 & 64,1 & - & Fuller et al 1974 \\
\hline Acide gras & 62,8 & 60,8 & $58, \mathbf{0}$ & Le Dividich et al 1987 \\
Insaturés (\%) & 57,4 & - & $53, \mathbf{6}$ & Lefaucheur et al 1989 \\
\hline
\end{tabular}

L'indice d'iode est une mesure du degré d'insaturation des graisses : plus il est élevé, plus elles sont insaturées.

cheur et al 1989, Rinaldo 1989), la taille des extrémités (queue, oreilles) est réduite et la pilosité plus importante. En outre, pour un même état d'engraissement, les résultats de Verstegen et al (1985) et Le Dividich et al (1987) suggèrent un effet de la température ambiante sur la répartition de la masse adipeuse : davantage de gras externe au froid et interne au chaud. Cette « redistribution » est liée, au chaud, d'une part, à une diminution de la lipogénèse qui est particulièrement marquée dans le tissu adipeux externe et, d'autre part, à une plus forte activité de la lipoprotéine lipase dans le tissu adipeux interne entraînant une captation plus importante de triglycérides dans ce tissu (Rinaldo 1989).

Ces modifications morphologiques et métaboliques sont le reflet d'une adaptation au milieu climatique. Il est enfin intéressant de noter une réduction du poids des organes, et notamment du foie, du coeur, et du tube digestif, avec l'augmentation de la température (Lefaucheur et al 1989), ce qui a pour conséquence d'améliorer le rendement en carcasse.

Pour ce qui concerne les caractéristiques qualitatives des tissus, l'influence de la température ambiante se manifeste notamment sur la composition en acides gras du tissu adipeux externe. Il est en effet établi qu'il existe une relation inverse entre le degré d'insaturation des dépôts adipeux sous-cutanés et la température ambiante (tableau 3). L'indice d'iode (Fuller et al 1974) ou le pourcentage d'acides gras insaturés (Le Dividich et al 1987, Lefaucheur et al 1989) diminuent avec l'augmentation de la température ambiante. Le lard des porcs maintenus à une température élevée est donc plus ferme et présente une meilleure aptitude à la conservation et aux transformations technologiques.

\section{4 / Température optimale}

Nous avons rassemblé dans la figure 4 les principaux critères d'estimation de la température optimale en prenant comme modèle le porc de 10 à $30 \mathrm{~kg}$ de poids vif élevé individuellement et nourri à volonté (Rinaldo et Le Dividich 1991). Il est clair que l'optimum thermique dépend de l'objectif recherché et, par conséquent, du critère considéré. Ainsi, par exemple, l'indice de consommation est minimal dans la zone de thermoneutralité, entre 25 et $31,5^{\circ} \mathrm{C}$ tandis que l'utilisation de l'énergie est maximale à la température critique $\left(25^{\circ} \mathrm{C}\right)$. Toutefois, dans la mesure où l'obtention d'une carcasse maigre et d'un coût alimentaire réduit sont des objectifs prioritaires, les résultats indiquent qu'en alimentation à volonté, la température optimale est supérieure à la température critique tout en demeurant dans la zone de neutralité thermique. Comme nous l'avons déjà souligné, elle ne correspond donc pas à une utilisation maximale de l'énergie. Mais, à cette température, il convient d'accepter une réduction, toutefois limitée, de la vitesse de croissance. Pour le porc en croissance-finition élevé en groupe sur caillebotis béton intégral, cette température optimale est de $24-25^{\circ} \mathrm{C}$, ce qui est notablement supérieur aux $18-21^{\circ} \mathrm{C}$ préconisés par le NRC (1981).

\section{La température ambiante influence l'état \\ d'engraissement \\ des animaux, la répartition et la composition en acides gras des lipides déposés.}


Figure 4. Influence de la température ambiante sur les performances de croissance, l'utilisation de l'énergie et la teneur en lipides du gain de poids vif vide du porc de 10 à $30 \mathrm{~kg}$ alimenté à volonté : signification d'une température optimale (d'après Rinaldo et Le Dividich 1991).

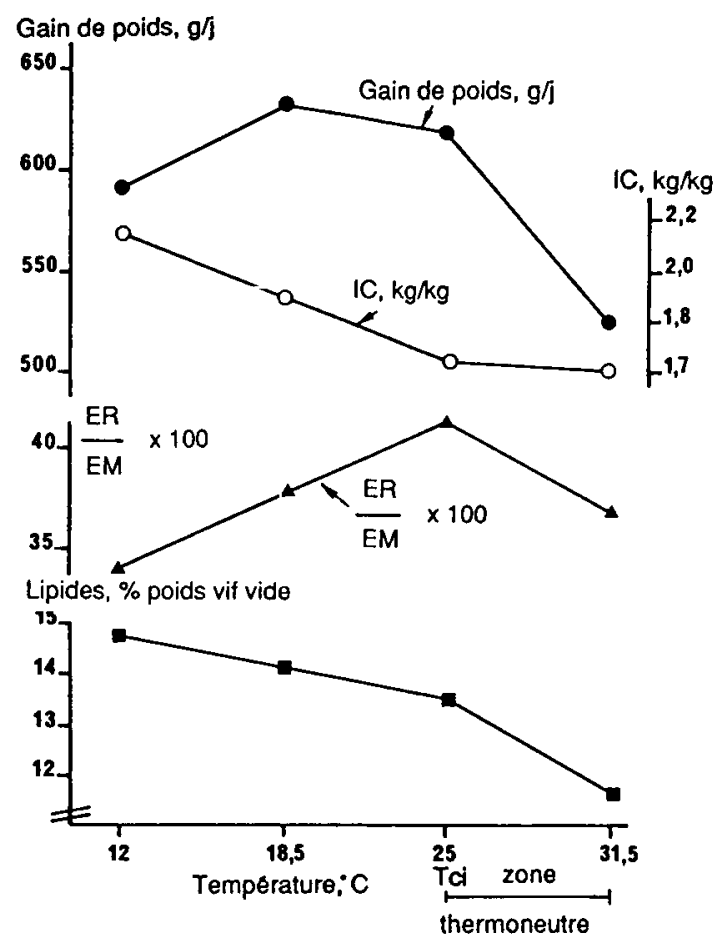

\section{3 / Interactions alimentation - température ambiante}

\section{1 / Apport d'énergie, de protéines et d'acides aminés}

\section{Pour un même gain de poids ou de muscle, la quantité totale de lysine ingérée est indépendante de la température ambiante.}

Tableau 4. Influence du taux de lysine de l'aliment sur les performances du porc alimenté à volonté, en relation avec la température ambiante (d'après Stahly et Cromwell 1987). décroît de $1^{\circ} \mathrm{C}$ dans l'intervalle $25-20^{\circ} \mathrm{C}$, ce besoin s'élève en moyenne à $12 \mathrm{~kJ} \mathrm{EM} / \mathrm{kg}^{0.75} / \mathrm{j}$; la valeur correspondante dans l'intervalle 20$12^{\circ} \mathrm{C}$ est de l'ordre de $25 \mathrm{~kJ} \mathrm{EM} / \mathrm{kg}^{0,75} / \mathrm{j}$ (Le Dividich et al 1985, 1987). Concrètement, pour un porc pesant $60 \mathrm{~kg}$, ceci représente 20 et $42 \mathrm{~g}$ d'aliment/" $\mathrm{C} /$ jour respectivement.

En alimentation à volonté, il existe une interaction entre le taux de protéines, et notamment le taux d'acides aminés indispensables, et la température ambiante sur les performances de croissance. Cette interaction repose sur le fait que l'augmentation de la consommation d'aliment avec l'abaissement de la température ambiante permet de compenser, selon le degré de déficit, un taux de protéines ou d'acides aminés qui s'avère insuffisant en conditions de thermoneutralité. Ainsi, un taux suboptimal de protéines (Seymour et al 1964) ou de lysine (Stahly et Cromwell 1987) a des répercussions moins importantes sur les performances du porc élevé au froid relátivement à la thermoneutralité (tableau 4). A même niveau alimentaire, nos résultats (Rinaldo 1989) indiquent que l'augmentation du taux de lysine améliore le gain de poids journalier d'autant plus que la température est plus élevée en raison d'une plus grande disponibilité de l'énergie pour la croissance. En revanche, lorsque les dépenses de thermorégulation sont couvertes par un apport correspondant d'énergie, les résultats présentés dans le tableau 5 montrent que pour une même quantité de lysine ingérée, les performances (gain de poids, composition corporelle, sont semblables au froid $\left(12^{\circ} \mathrm{C}\right)$ et à la thermoneutralité $\left(20^{\circ} \mathrm{C}\right)$.

En définitive, seul le besoin énergétique dépend de la température ambiante. En revanche, le besoin azoté pour un même gain de poids ou de muscle est indépendant de l'environnement thermique lorsque l'apport énergétique n'est pas limitant. Toutefois, relativement à l'énergie, il augmente avec la température ambiante par suite d'une plus grande disponibilité de l'énergie pour les synthèses. Selon nos résultats (Rinaldo 1989), à $1^{\prime \prime} \mathrm{C}$ d'augmentation de la température ambiante entre 12 et $20^{\circ} \mathrm{C}$, correspond un accroissement du besoin en lysine de 0,009 g/MJ EM ; entre 20 et $25^{\prime \prime} \mathrm{C}$, l'accroissement est de 0,005 g/MJ EM.

\section{2 / Concentration en énergie du régime}

L'utilisation de matières premières riches en constituants cellulosiques et en matières

\begin{tabular}{|l|c|c|c|}
\hline & \multirow{2}{*}{$\begin{array}{c}\text { Température } \\
\text { ambiante }\end{array}$} & \multicolumn{2}{|c|}{ Lysine, $^{\mathbf{0}}$} \\
\cline { 3 - 4 } & & $\mathbf{0 , 5 0}$ & $\mathbf{0 , 9 5}$ \\
\hline Gain de poids/j & 10 & 95 & 100 \\
& 22,5 & 86 & 100 \\
Indice de consommation & 10 & 107 & 100 \\
& 22,5 & 114 & 100 \\
Gain de muscle/j & 10 & 91 & 100 \\
& 22,5 & 86 & 100 \\
\hline
\end{tabular}

1 - A chaque température la valeur 100 est attribuée aux performances obtenues avec le taux de $0,95 \%$ de lysine. 


\begin{tabular}{|c|c|c|c|c|c|}
\hline \multirow{2}{*}{$\begin{array}{c}\text { Température ambiante, }{ }^{\circ} \mathrm{C} \\
\text { Lysine } \% \text {, Croissance } \\
\text { Finition }\end{array}$} & \multicolumn{2}{|c|}{12} & \multicolumn{2}{|c|}{20} & \multirow[b]{2}{*}{$S x^{1}$} \\
\hline & $\begin{array}{l}\mathbf{0 , 6 5} \\
\mathbf{0 , 5 5}\end{array}$ & $\begin{array}{l}\mathbf{0 , 8 0} \\
0,70\end{array}$ & $\begin{array}{l}\mathbf{0 , 6 5} \\
\mathbf{0 , 5 5}\end{array}$ & $\begin{array}{l}\mathbf{0 , 8 0} \\
\mathbf{0 , 7 0}\end{array}$ & \\
\hline $\begin{array}{l}\text { Aliment ingéré, g/j } \\
\text { Régime de base }{ }^{2} \\
\text { Amidon de maïs }\end{array}$ & $\begin{array}{c}2198 \\
268\end{array}$ & $\begin{array}{c}2200 \\
273\end{array}$ & 2231 & 2220 & \\
\hline Gain de poids, $g / j$ & 738 & 770 & 730 & 776 & 7,$0 ; \mathrm{L}^{* *}$ \\
\hline Indice de consommation & 3,34 & 3,21 & 3,06 & 2,86 & 0,$03 ; \mathrm{L}^{* *} \mathrm{~T}^{* *}$ \\
\hline Lysine ingérée, g/MJ EM & 0,40 & 0,50 & 0,47 & 0,58 & \\
\hline Caractéristiques des carcasses & & & & & \\
\hline Pourcentage de gras & 28,4 & 27,6 & 28,2 & 28,5 & 2,9 \\
\hline Pourcentage de muscle & 48,3 & 50,1 & 48,6 & 49,3 & 2,4 \\
\hline
\end{tabular}

1 - Ecart-type de la moyenne ; L, effet lysine; T, effet température, ${ }^{* *}, \mathrm{P}<0,01$.

2 - Régime à base de blé et de tourteau de soja à $16 \%$ de protéines brutes en période de croissance et $13 \%$ en période de finition.

Tableau 6. Influence de la température ambiante et de la concentration en énergie de l'aliment sur la production de chaleur et la partition de l'énergie fixée $\left(\mathrm{kJ} / \mathrm{kg}^{0,75} / \mathrm{j}\right)$ chez le porc en croissance (d'après Noblet et al 1985) (Données ajustées à une même quantité d'EM ingérée, soit $1323 \mathrm{~kJ} / \mathrm{kg}^{0,75} / \mathrm{j}$ ).

\begin{tabular}{|l|c|c|c|c|}
\hline Température ambiante, $^{\mathbf{}} \mathbf{C}$ & \multicolumn{2}{|c|}{13} & \multicolumn{2}{c|}{$\mathbf{2 3}$} \\
\hline \multicolumn{1}{|c|}{ Aliment $^{1}$} & $\mathrm{C}$ & $\mathrm{D}$ & $\mathrm{C}$ & $\mathrm{D}$ \\
\hline Production de chaleur & 797 & 809 & 663 & 759 \\
Energie fixée & 526 & 514 & 660 & 564 \\
Protéines fixées & 128 & 127 & 174 & 186 \\
Lipides fixés & 398 & 387 & 486 & 378 \\
\hline
\end{tabular}

1 - C, aliment concentré en énergie ; D, aliment dilué en énergie.

grasses par le porc est, dans une certaine mesure, liée à la température ambiante par le biais de l'extra-chaleur qui leur est associée. Cette dernière participe aux dépenses de thermorégulation chez l'animal exposé au froid (Verstegen et al 1973), alors qu'en conditions de thermoneutralité elle est obligatoirement évacuée. Par ailleurs, son importance dépend notamment de la teneur en constituants cellulosiques et en matières grasses du régime. Les travaux de Just (1982) montrent, à cet égard, une augmentation de la production d'extra-chaleur avec le taux de cellulose brute du régime qui s'explique à la fois par la chaleur associée à la dégradation microbienne de la cellulose dans le gros intestin et par le faible rendement des produits terminaux de la digestion (A.G.V.) pour les synthèses. A l'inverse, la production d'extra-chaleur est faible pour les aliments riches en matières grasses qui se déposent avec un minimum de remaniements biochimiques. Il en ressort que l'utilisation par le porc de régimes peu concentrés en énergie par incorporation de matières premières riches en constituants cellulosiques serait ainsi améliorée au froid relativement aux aliments fortement concentrés.

Les résultats obtenus sur le porcelet et le porc en croissance-finition nourris à volonté font état d'une interaction entre la température ambiante et la concentration en énergie des régimes sur les performances de croissance (Coffey et al 1982, Le Dividich et Noblet 1986). Au froid, ces performances sont relativement indépendantes de la concentration en énergie des aliments, alors qu'au chaud, elles sont améliorées par l'utilisation de rations concentrées en énergie par addition de matières grasses (figure 5). La similitude des performances obtenues au froid avec les régimes dilués et concentrés en énergie est dûe essentiellement au fait que la quantité d'énergie digestible ingérée ne varie pas avec la concentration énergétique de l'aliment. En outre, les travaux de Noblet et al (1985) montrent une participation de l'extrachaleur liée à l'utilisation des régimes dilués à la thermorégulation (tableau 6). En effet, relativement à la thermoneutralité $\left(23^{\circ} \mathrm{C}\right)$, l'augmen-

Figure 5. Influence de la saison ou de la température ambiante et de la concentration en énergie de l'aliment sur les performances du porc en croissance $(A)$ et du porcelet $(B)$ (d'après Coffey et al 1982 (A) et Le Dividich et Noblet 1986 (B)).
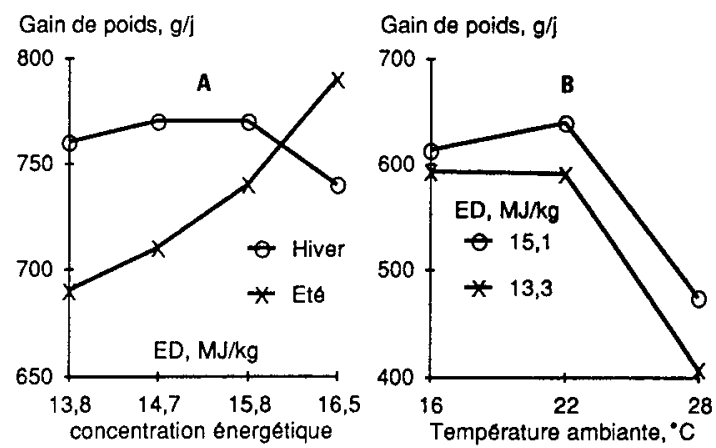

Tableau 5. Effet de la température ambiante et du taux de lysine de la ration sur les performances de croissance du porc lorsque le "froid" est compensé par un apport supplémentaire d'énergie (d'après Rinaldo 1989). 
tation de la production de chaleur au froid $\left(13^{\circ} \mathrm{C}\right)$ est moins élevée avec un régime dilué qu'avec un régime concentré en énergie. Néanmoins, l'énergie ainsi épargnée est intégralement fixée sous forme de lipides, augmentant l'adiposité des carcasses. En revanche, au chaud, les meilleures performances obtenues avec des rations enrichies en matières grasses sont liées à l'augmentation de la consommation d'énergie digestible.

En définitive, on peut supposer une meilleure valorisation au froid des aliments peu concentrés en énergie. Néanmoins, si l'on tient compte de leur effet sur l'état d'engraissement des animaux et de la diminution de 1 à 2 points du rendement en carcasse, il est clair que l'utilisation en période froide de régimes riches en constituants cellulosiques a un intérêt limité chez le porc en croissance. Malgré tout, en période hivernale, l'emploi de ces régimes s'avère intéressant chez la truie en gestation (Noblet et al 1988).

\section{Conclusion}

En conclusion, il est intéressant de souligner l'importance des conditions climatiques de logement sur les performances de croissance du porc et l'économie de la production. A $1^{\circ} \mathrm{C}$ d'augmentation de la température ambiante entre 10 et $20^{\circ} \mathrm{C}$ correspond une économie d'environ $3,3 \mathrm{~kg}$ d'aliment par porc produit ; entre 20 et $24-25^{\circ} \mathrm{C}$ une économie de 1,2 à $1,4 \mathrm{~kg}$ peut être escomptée. La maîtrise des conditions d'environnement thermique peut donc contribuer, de manière importante, à la réduction des coûts de production et notamment du coût alimentaire. Si l'on ajoute à cela que le rendement en carcasse varie dans le même sens que la température ambiante, on a intérêt, dans les conditions actuelles d'élevage sur caillebotis à maintenir dans les bâtiments une température ambiante élevée $\left(24-25^{\circ} \mathrm{C}\right)$. En période hivernale, l'intérêt d'un chauffage artificiel dépend des coûts relatifs de l'énergie thermique et alimentaire. Mais, avant tout, il est essentiel de conserver la chaleur dissipée par les animaux grâce notamment à l'isolation thermique des locaux et à un renouvellement adéquat de l'air. Dans ce domaine, il est donc important de connaître les effets des constituants de l'air, notamment de l'hygrométrie et de certains gaz nocifs comme l'ammoniac, sur les performances et la santé des animaux afin de définir un taux minimum de renouvellement de l'air. Par ailleurs, compte tenu de l'influence prépondérante de la température ambiante sur la consommation et l'utilisation métabolique de l'énergie, il est nécessaire de poursuivre les travaux sur le réajustement des apports nutritionnels, en protéines et acides aminés notamment, dans les aliments du porc placé dans les conditions climatiques optimales.

\section{Références bibliographiques}

BERSCHAUER F., CLOSE W.H., STEPHENS D.B., 1983. The influence of protein/energy value of the ration and level of feed intake on the energy and nitrogen metabolism of the growing pig. 2- N metabolism at 2 environmental temperatures. Br. J. Nutr., 49, 271-283.

BRESK B., STOLPE J., 1982. Fattening performance tests for assessement of critical temperature of swine. Mh. vet.-Med. 37, 374-380.

CAMPBELL R.G., TAVERNER M.R., 1988. Relationships between energy intake and protein and energy metabolism, growth and body composition of pigs kept at 14 or $32^{\circ} \mathrm{C}$ from 9 to $20 \mathrm{~kg}$. Livest. Prod. Sci., 18, 289-303.

CLOSE W.H., 1978. The effects of plane of nutrition and environmental temperature on the energy metabolism of the growing pig. 3- The efficiency of energy utilization for maintenance and growth. Br. J. Nutr., 40, 433-438.

CLOSE W.H., 1981. The climatic requirements of the pig. In Environmental aspects of housing for animal production, CLARK J.A. (Ed.), Butterworths, London, 149-166.

CLOSE W.H., MOUNT L.E., BROWN D., 1978. The effects of place of nutrition and environmental temperature on the energy metabolism of the growing pig. 2- Growth rate, including protein and fat deposition. Br. J. Nutr., 40, 423-431.

COFFEY M.T., SEERLEY R.W., FUNDERBUKE D.W., MC CAMPBELL H.C., 1982. Effect of heat increment and level of dietary energy and environmental temperature on the performance of growing-swine. J. Anim. Sci., 54, 95105 .

FULLER M.F., BOYNE A.W., 1971. The effects of environmental temperature on the growth and metabolism of pigs given different amounts of food. 1- Nitrogen metabolism, growth and body composition. Br. J. Nutr., 25, 259272 .

FULLER M.F., BOYNE A.W., 1972. The effects of environmental temperature on the growth and metabolism of pigs given different amounts of food. 2- Energy metabolism. Br. J. Nutr. 28, 373-384.
FULLER MF, DUNCAN WRH, BOYNE A.W, 1974 Effect of environmental temperature on the degree of unsaturation of depot fats of pigs given different amounts of food. J. Sci. Food. Agric., 25, 205-210.

HERPIN P., 1988. Mécanismes et régulation de la thermogénèse chez le jeune porc exposé au froid. Thèse de Docteur-Ingénieur en Sciences Agronomiques, INA Paris-Grignon, $131 \mathrm{pp}$.

JUST A., 1982. The net energy value of balanced diets for growing pigs. Livest. Prod. Sci., 8, 541-555.

LE DIVIDICH J., NOBLET J., 1986. Effect of dietary energy level on the performance of individually housed early-weaned piglets in relation to environmental temperature. Livest. Prod. Sci., 14, 255-263.

LE DIVIDICH J., VERMOREL M., NOBLET J., BOUVIER J.C., AUMATTRE A., 1980. Effects of environmental temperature on heat production, energy retention, protein and fat gain in early-weaned piglets. Br. J. Nutr., 44, 313323.

LE DIVIDICH J., NOBLET J., AUMAITRE A., 1982. Environmental requirements of early-weaned intensively reared piglets. In Proceedings of the 2nd International Livestock Fnvironment Symposium, April 20-23, 1982 A.S.A.E. (Ed.), Urbana, Illinois, 353-361.

LE DIVIDICH J., DESMOULIN B., DOURMAD JY. 1985. Influence de la température ambiante sur les performances du porc en croissance-finition en relation avec le niveau alimentaire. J. Rech. Porcine en France, 17, 275282 .

LE DIVIDICH J., NOBLET J., BIKAWA T., 1987. Effect of environmental temperature and dietary energy concentration on the performance and carcass characteristics of growing-finishing swine fed to equal rate of gain. Livest. Prod. Sci., 17, 235-246.

LEFAUCHEUR L., LE DIVIDICH J., KRAUSS D., ECOLAN P., MOUROT J., MONIN G., 1989. Influence de la température d'élevage sur la croissance, le métabolisme musculaire, et la qualité de la viande. Journées Rech. Porcines en France, 21, 231-238. 
NICHOLS D.A., AMES D.R., HINFS R.H., 1982. Effect of temperature on performance and efficiency of finishing swine. Proceedings of the 2nd International Livestock Environment Symposium April 20-23, 1982, Urbana, Illinois, ASAE (Ed.), 376-379.

NIENABER I.A., LE ROY HAHN G., 1983. Performance of growing-finishing swine in response to the thermal environment. ASAE Mid-Central meeting, March 16, 1983 St-Joseph, Missouri, paper n" MCR 83-137.

NOBLET J., LE DIVIDICH J.. 1982. Effect of environmental temperature and feeding level on energy balance traits of early-weaned piglets. Livest. Prod. Sci., 9, 619-632.

NOBLET J., LE DIVIDICH J., BIKAWA T., 1985. Interaction between energy level in the diet and environmenta temperature on the utilization of energy in growing pigs. J. Anim. Sci., 61, 452-459.

NOBLET J., DOURMAD J.Y.. DUBOIS S., LE DIVIDICH J. 1988. Influence de la température ambiante sur les dépenses énergétiques de la truie gravide. Interaction avec la nature du régime (paille, luzerne). Journées Rech. Porcines en France, 20, 345-350.

N.R.C., 1981. Effect of environment on nutrient requirements of domestic animals. Nat'l Research Council Nat'l Academy of Sciences, Washington D.C., $152 \mathrm{pp}$.

RINALDO D., 1989. Influence de la température ambiante sur le métabolisme énergétique el tissulaire et le besoin en lysine du porc en croissance. Mise en évidence de l'intérêt d'une température élevée. Thèse de l'Université de Rennes I, $124 \mathrm{pp}$.

RINALDO D., LE DIVIDICH J., 1991. Assessment of optimal temperature for performance and chemical body composition of growing pigs. Livest. Prod. Sci. (sous presse)
RINALDO D., SALAUN M.C., LE DIVIDICH J., 1989. Influence d'une réduction de la température ambiante ou d'un abaissement nocturne de la température ambiante sur les performances du porcelet sevré. Journées Rech. Porcine en France, 21, 239-244.

SEYMOUR E.W., SPEER V.C., HAYS V.W., MANGOLD D.W. HAZEN T.E., 1964. Effects of dietary protein level and environmental temperature on performance and carcass quality of growing-finishing swine. J. Anim. Sci., 23, 375-379.

STAHLY T.S., CROMWELL G.L., 1987. Optimal dietary Iysine levels for pigs as influenced by the thermal environment. Swine Research Report, University of Kentucky, Departement of Anim. Sci., Lexington, Report n"299, 15 18.

THOMAS P., 1984. The influence of housing design and some management systems on the health of the growing pig, particularly in relation to pneumonia. Pig News Inf., 5, $343-349$

VERSTEGEN M.W.A., CLOSE W.H., START I.B., MOUNT L.E., 1973. The effects of environmental temperature and plane of nutrition on heat loss, energy retention and deposition of protein and fat in groups of growing pigs. Br. J. Nutr., 30, 21-35.

VERSTEGEN M.W.A., BRASCAMP E.W., VAN DER HEL W. 1978. Growing and fattening of pigs in relation to temperature of housing and feeding level. Can. J. Anim. Sci., 58, 1-13.

VERSTEGEN M.W.A., BRANDSMA H.A., MATEMAN G., 1985. Effect of ambient temperature and feeding level on slaughter quality in fattening pigs. Neth. J. Agric. Sci., 33, 1-15.

\section{Summary}

Influence of environmental temperature on growth performance in pigs.

The aim of the present paper is to present the effects of environmental temperature on energy balance, growth performance and nutritional requirements of growing-finishing swine and to assess the optimal temperature. The critical temperature is estimated at to about $20^{\circ} \mathrm{C}$ for growing pigs and to $15^{\circ} \mathrm{C}$ for finishing ones. The critical temperature corresponds to a maximal utilization of feed energy and its significance is discussed in terms of animal performance. Environmental temperature mainly affects fat deposition. In ad-libitum conditions, volontary feed intake decreases by $22 \mathrm{~g} / \mathrm{d} /{ }^{\circ} \mathrm{C}$ between 10 and $20^{\circ} \mathrm{C}$, whithout any effect on daily weight gain. Between 20 and $30^{\circ} \mathrm{C}$, this decrease is more pronounced $\left(73 \mathrm{~g} / \mathrm{j} /{ }^{\circ} \mathrm{C}\right)$ and involves a reduc- tion in daily weight gain $\left(40 \mathrm{~g} /{ }^{\circ} \mathrm{C}\right)$ and in body fatness. On the basis of reduced production costs and body fatness, the optimal temperature for growing-finishing swine reared on concrete slatted floors is assessed at $24-25^{\circ} \mathrm{C}$. Studies on the interactions between temperature and nutritional requirements suggest that, in warm conditions, performance is improved in pigs fed high energy diets whereas, in the cold, growth is independent of feed energy concentration. The increase in energy requirement between 20 and $12^{\circ} \mathrm{C}$ is assessed at $25 \mathrm{~kJ} \mathrm{EM} / \mathrm{kg0.75} / \mathrm{d} /{ }^{\circ} \mathrm{C}$. The daily amount of essential amino acids required for a given weight gain or muscle gain does not vary with environmental temperature.

RINA LDO Dominique, LE DIVIDICH J., 1991. Influence de la température ambiante sur les performances de croissance du porc. INRA Prod. Anim., 4 (1), 57-65. 